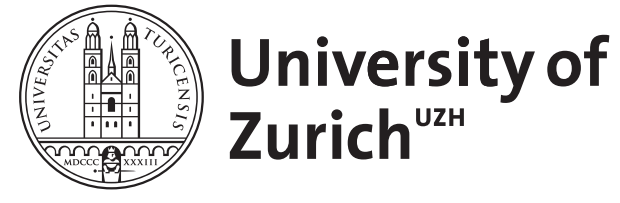

Zurich Open Repository and Archive

University of Zurich

University Library

Strickhofstrasse 39

CH-8057 Zurich

www.zora.uzh.ch

Year: 2014

Welche Ansprüche haben meine Patienten an mich und welche Wünsche stecken dahinter?

Dengler-Voss, Carolin ; Voss, Rödiger

Posted at the Zurich Open Repository and Archive, University of Zurich

ZORA URL: https://doi.org/10.5167/uzh-99461

Journal Article

Published Version

Originally published at:

Dengler-Voss, Carolin; Voss, Rödiger (2014). Welche Ansprüche haben meine Patienten an mich und welche Wünsche stecken dahinter? PrimaryCare, 14(18):289-291. 


\section{Welche Ansprüche haben meine Patienten an mich und welche Wünsche stecken dahinter?}

\begin{abstract}
Einleitung
Hausärzte haben täglich Umgang mit zahlreichen Patienten. Diese kommen unterschiedlich informiert und mit differenzierten Erwartungen und Ansprüchen gegenüber ihrem Arzt in die Praxis. Aufgrund der Komplexität und Langfristigkeit geht die Hausarzt-Patienten-Beziehung weit über ein reines Dienstleistungsmodell hinaus [1]. Die Erkenntnisse dieser Studie sollen helfen, die Beweggründe von Patienten verständlicher zu machen und die Beziehung zwischen Hausarzt und Patient zu optimieren. Es handelt sich um die erstmalige Anwendung der Laddering-Technik zur Ableitung der Patientenansprüche an ihren Hausarzt in der Schweiz.
\end{abstract}

\section{Methodik}

Die aus der amerikanischen Marktforschung bekannte LadderingTechnik wird in der vorliegenden Studie eingesetzt, um Beziehungen zwischen Leistungsansprüchen von Patienten an Hausärzte und die damit verbundenen persönlichen Werte aufzuzeigen. Beim «Laddern» wird darauf geachtet, dass eine Kette entsteht, ausgehend von einer Kompetenz bzw. Qualifikation von Ärzten über Konsequenzen (Nutzeneigenschaften), die mit der Kompetenz verbunden sind, bis hin zu dem Wert, der dahinter steht. Vor diesem Hintergrund wurden Patienten befragt, welche Kompetenzen und Qualifikationen Hausärzte in eine Konsultation einbringen sollten. Im Anschluss wurde eruiert, was der Nutzen der genannten Attribute ist («warum ist die genannte Eigenschaft/Fähigkeit wichtig») und welche Werte dahinterstehen. Dazu ein Beispiel, um ein Gespräch zwischen Interviewer (I) und Patienten (P) zu veranschaulichen:

\section{Tabelle 1}

Beispiel eines Laddering-Interviews.

I: Welche Kompetenzen und Qualifikationen würden Sie sich bei Hausärzten während einer Konsultation wünschen?

P: Fachwissen, Kommunikationsfähigkeiten ... entspricht Attributen.

I: Warum ist das Fachwissen eines Arztes für Sie wichtig?

P: Ich will gut behandelt werden. Der Arzt soll das Richtige feststellen ... Nutzen.

I: Warum wollen Sie gut behandelt werden?

P: Ich will schnell wieder fit sein. ... entspricht Nutzen

I: Warum wollen Sie schnell wieder fit sein?

P: Eine gute Gesundheit ist doch für jeden wichtig, oder? ... entspricht Wert

Software-unterstützt wurde aus den Daten inhaltsanalytisch eine sogenannte «Hierarchical Value Map» (HVM) generiert. Es handelt sich um eine graphische Darstellung der wichtigsten LadderingChains aller Befragten, um ihr Verhalten und ihre Wünsche zu verstehen und zu erklären.

\section{Resultate}

Es wurden insgesamt 35 Personen (19 weiblich, 16 männlich) in der Deutschschweiz befragt. Alle Interviewten waren berufstätig und zwischen 40 und 65 Jahren alt. Im Rahmen der theoretischen Sample-Bildung wurde diese Altersgruppe gewählt, weil Individuen in diesem Alter aufgrund ihrer Lebenserfahrung bereits ausreichend Eindrücke und Ansprüche im Umgang mit Ärzten gesammelt haben. Es wurden nur Personen ins Sample aufgenommen, die in den vergangenen zwei Jahren mindestens drei Kontakte zum Hausarzt hatten. Das Durchschnittsalter betrug rund 52 Jahre. Knapp 80\% der Befragten wiesen mehr als drei Hausarztkonsultationen jährlich auf.

Zur Interpretation der HVM: Die Grösse der Kreise steht für die Häufigkeit, mit der Attribute, Konsequenzen und Werte von Befragten genannt wurden. Die Breite der Verbindungslinien zwischen Attributen, Konsequenzen und Werten repräsentiert die Häufigkeit, mit der die jeweiligen Konzepte gemeinsam genannt wurden. In der HVM werden die Verbindungen zwischen Attributen (weisse Kreise), Konsequenzen (graue Kreise) und Werten (schwarze Kreise) dargestellt (vgl. Abb. 1).

Aus der HVM ist leicht ersichtlich, dass Patienten von Hausärzten sieben wesentliche Kompetenzen und Qualifikationen erwarten: Fachwissen, Kommunikationskompetenz, Freundlichkeit, Empathie, proaktiv sein, Berufserfahrungen und Geduld. Dies sind keine gravierend innovativen Studienergebnisse für die Ansprüche von Patienten. In Vergleichsstudien aus der Schweiz [2], England [3] und Deutschland [4] wurden affine Attribute ermittelt. Besonders interessant sind die Verbindungen zu Patientennutzen und Werten, die im Folgenden neben den Attributen selbst diskutiert werden.

\section{Diskussion}

Das Fachwissen eines Allgemeinmediziners ist in unserer Umfrage das vorherrschende Attribut. Schläpfer [2] dokumentiert im Rahmen einer repräsentativen Umfrage in der Schweiz ebenso diesen besonderen Stellenwert. Eine gründliche Ausbildung sowie Fortund Weiterbildungen sind aus diesem Grund ein wichtiger Bestandteil der ärztlichen Tätigkeit. Zum Fachwissen zählt für die befragten Patienten auch der Aspekt, an einen entsprechenden Spezialisten weiterzuweisen zu können. Die Probanden sehen den Hausarzt demnach nicht als «Alleskönner». Ein Proband erklärte beispielsweise, dass das vorhandene Fachwissen spürbar sei, wenn die Behandlung reibungslos verlaufe. Die Wahrnehmung der ärztlichen Kompetenz hat weitreichende Folgen: Wenn ein Patient von der Kompetenz des Arztes überzeugt ist, nimmt er selbst die Unfreundlichkeit der medizinischen Praxisassistentinnen in Kauf, auch wenn dieses Verhalten ihn erheblich stört [6]. Allgemeinmediziner können nach Ansicht unserer Probanden ihrer Kompetenz Ausdruck verleihen, indem sie sich auf aktuelle Forschungsergebnisse beziehen. Die jüngeren Probanden in unserem Kollektiv schauten sich auch die Internetpräsenz ihrer Ärzte an sowie deren dokumentierten Lebenslauf. Es lohnt sich also für den Allgemeinmediziner, 


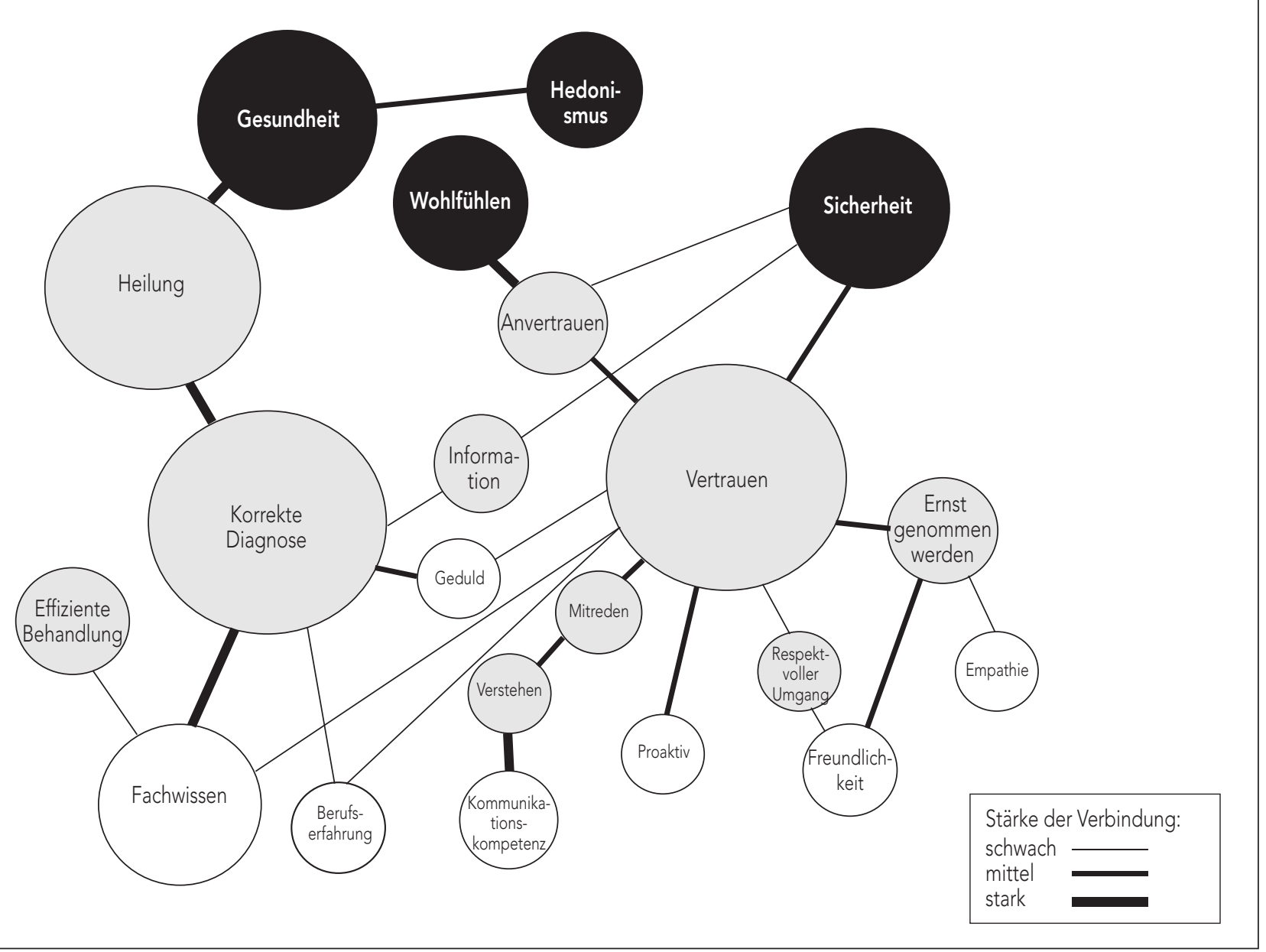

Abbildung 1

Patienten-«Hierarchical Value Map» (HVM).

hier präsent zu sein. Daneben können in der Praxis aufgehängte Weiterbildungszertifikate das aktuelle Fachwissen ausweisen.

Durch sein Fachwissen kann der Arzt nach Meinung der Patienten eine effiziente Behandlung ermöglichen, was vor allem zeiteffizientes Arbeiten beinhaltet. Ungern warten Patienten lange im Wartezimmer auf einen vereinbarten Termin. Eine langjährige Berufserfahrung eines Hausarztes wirkt ähnlich wie das Fachwissen. Studienergebnisse von Neuenschwander/Riedel [1] identifizieren eine langjährige Erfahrung als Kriterium eines guten Hausarztes. Berufserfahrung und Fachwissen helfen nach Meinung der Probanden bei der Feststellung der korrekten Diagnose und damit der richtigen Behandlung, was zur Genesung und dann zum Wert Gesundheit führt.

Bei der Konsultation wird vom Allgemeinmediziner eine gute Kommunikationsfähigkeit erwartet. Die Patienten wollen die Schilderungen des Arztes verstehen: Ihrer Meinung nach sollte der Gebrauch von ärztlichem Fachjargon vermieden werden und Erklärungen auf verständliche Art und Weise erfolgen. Lateinische Fachausdrücke, wirken kontraproduktiv, da dem Patienten diese Spezialausdrücke im Sprachgebrauch fehlen. Peters [6] betont, dass gesprächslinguistische Studien zeigen, dass eine gute Kommunikation zu einer besseren Compliance führt. Die Patienten wollen die ärztlichen Entscheidungen nicht nur verstehen, sondern diese auch mit dem Allgemeinmediziner besprechen. Letzterer sollte daher Nachrichten deuten und Meinungen verstehen kön- nen. Ärzte können diese Fähigkeiten durch Rollenspiele und weitere Übungsmethoden mit erfahrenen Kollegen, Kommunikationstrainern oder speziellen Weiterbildungsangeboten [7] gewinnen oder verbessern.

Ein weiteres Attribut eines Hausarztes sollte Geduld sein, d.h. er soll sich für die Patienten und deren Behandlung Zeit nehmen. Dadurch erkennt er, was das Anliegen seiner Patienten ist und er kann zu einer korrekten Diagnose kommen. Zudem begünstigt die Geduld die Vertrauensbildung der Patienten, was folgende Aussage einer Patientin belegt: «Wenn er sich Zeit nimmt, ist er an mir interessiert, dem vertraue ich dann.»

Freundlichkeit ist neben dem Fachwissen und der Kommunikationskompetenz für die Patienten eines der drei wichtigsten Attribute und somit ein Kernaspekt einer respektvollen Behandlung. Wenn ein Arzt freundlich ist und zudem Empathie zeigt, fühlen sich die Patienten ernst genommen. Forschungsergebnisse von Neuenschwander und Riedel [1] belegen ebenso, dass sich der Arzt Zeit für Patienten und diese ernst nehmen soll. Dadurch sehen Patienten Ärzte als Partner in einer gleichberechtigten Beziehung. Als Resultat daraus finden Patienten zu ihrem Arzt Vertrauen und können sich ihm gegenüber öffnen («Anvertrauen»), was ein Gefühl des Wohlfühlens entstehen lässt.

Weniger stark ausgeprägt ist das Attribut Proaktivität. Darunter sind das aktive Nachfragen des Arztes sowie sein gezeigter Einsatz zusammenzufassen, d.h. Vorstellungen und Erwartungen der Pa- 
tienten sollen aktiv erfragt und an einem gemeinsamen Vorgehen Interesse gezeigt werden. Donner-Banzhoff [8] fasst diese Kriterien unter dem patientenzentrierten Vorgehen von Ärzten zusammen.

\section{Hauptaussage}

Patienten erhoffen, dass durch Fachwissen, Geduld und Erfahrung ihres Hausarztes eine möglichst zutreffende Diagnose gestellt wird. Ziel ist für den Patienten die Heilung und letztlich die Gesundheit. Gesundheit ist einer der zentralen Werte der Patienten. Eine solche Fokussierung war zu prognostizieren, da das primäre Behandlungsziel die Verbesserung des Gesundheitszustandes des Patienten ist. Individuen möchten nach Krankheit oder Unfall schnellstmöglich wieder in ihr normales Leben zurückfinden und Zeit mit ihren Familien und ihrem sozialen Umfeld verbringen und geniessen können. Im gesunden Zustand können Individuen nach Freude, Genuss und Lust (Hedonismus) streben. Des Weiteren gibt eine richtige Diagnose die Möglichkeit, den Patienten über seinen Zustand zu informieren, was ihm ein Gefühl von Sicherheit bringt. Patienten erwarten nach der Diagnose eine ausreichende und umfassende Information durch den Hausarzt.

Vertrauen ist neben der korrekten Diagnose die zentrale Nutzeneigenschaft der Patienten. Wenn die Patienten Vertrauen entwickeln, können sie sich öffnen und frei mitteilen, auch wenn es sich um sehr intime Gegebenheiten handelt. Die Relevanz des Vertrauens ist im Kontakt zwischen Arzt und Patient wie folgt zu erklären: Im Zeitpunkt der Anamnese befindet sich der Patient durch körperliches und/oder seelisches Leid in einer geschwächten Position. Damit sich ein Patient in dieser Lage öffnet und seinem Arzt auch zu heiklen Fragen ehrlich Auskunft gibt, braucht es Vertrauen. Für die Vertrauensgewinnung sind alle abgeleiteten Attribute relevant, also, Fachwissen, Freundlichkeit, Berufserfahrung, Empathie, pro- aktives Verhalten und Geduld. Alle genannten Attribute besitzen eine direkte oder indirekte Verbindung zum Vertrauen. Erst wenn Patienten dem Arzt Vertrauen und sich ihm öffnen können, entwickeln sie ein Gefühl des Wohlempfindens und der Sicherheit.

\section{Literatur}

1 Neuenschwander P, Riedel M. Was Patienten über ihren Hausarzt wissen wollen: eine qualitative Studie. Schweizerische Ärztezeitung. 2009;90:698700.

2 Schläpfer L. Hausärzte, wie Patienten sie sich wünschen. Schweizerische Ärztezeitung. 2010;91:1022-3.

3 Gruber T, Frugone F. Uncovering the desired qualities and behaviours of general practitioners (GPs) during medical (service recovery) encounters. Journal of Service Management. 2011;22:491-521.

4 Dieterich A. Arzt-Patient-Beziehung im Wandel: Eigenverantwortlich, informiert, anspruchsvoll. Deutsches Ärzteblatt. 2007;104:2489.

5 Letter K. Praxisführung: Den Patienten als Kritiker akzeptieren. Deutsches Ärzteblatt. 2010;107:1039-40.

6 Peters T. Macht im Kommunikationsgefälle: der Arzt und sein Patient. Band 82. Berlin: Frank \& Timme GmbH Verlag; 2008.

7 Diviani-Preiswerk S. Kommunikation in der Praxis: Wie sag ich's meinen Patienten? Schweizerische Ärztezeitung. 2014;95:616-8.

8 Donner-Banzhoff N. Arzt und Patient: Archäologie einer Beziehung. Deutsches Ärzteblatt. 2012;109:2078.

\section{Korrespondenz:}

Dr. med. Carolin Dengler-Voss

Fachärztin FMH für Allgemeine Innere Medizin

Verkehrsmedizinerin SGRM

Universität Zürich, Institut für Rechtsmedizin -

Abteilung Verkehrsmedizin \& forensische Psychiatrie

carolin.dengler-voss[at]irm.uzh.ch 